\title{
Searching for X-ray emission from AGB stars ${ }^{\star}$ (Research Note)
}

\author{
S. Ramstedt ${ }^{1}$, R. Montez ${ }^{2}$, J. Kastner ${ }^{2}$, and W. H. T. Vlemmings ${ }^{3}$ \\ 1 Argelander Institute for Astronomy, University of Bonn, 53121 Bonn, Germany \\ e-mail: sofia@astro.uni-bonn.de \\ 2 Rochester Institute of Technology, 54 Lomb Memorial Drive, Rochester, NY 14623, USA \\ 3 Onsala Space Observatory, 43992 Onsala, Sweden
}

Received 24 November 2011 / Accepted 18 June 2012

\section{ABSTRACT}

\begin{abstract}
Context. Magnetic fields have been measured around asymptotic giant branch (AGB) stars of all chemical types using maser polarization observations. If present, a large-scale magnetic field would lead to X-ray emission, which should be observable using current X-ray observatories.

Aims. The aim is to search the archival data for AGB stars that are intrinsic X-ray emitters.

Methods. We have searched the ROSAT, CXO, and XMM-Newton archives for serendipitous X-ray observations of a sample of $~ 500$ AGB stars. We specifically searched for the AGB stars detected with GALEX. The data is calibrated, analyzed and the $\mathrm{X}$-ray luminosities and temperatures are estimated as functions of the circumstellar absorption.

Results. We identify 13 AGB stars as having either serendipitous or targeted observations in the X-ray data archives, however for a majority of the sources the detailed analysis show that the detections are questionable. Two new sources are detected by ROSAT: T Dra and R UMa. The spectral analysis suggests that the emission associated with these sources could be due to coronal activity or interaction across a binary system.

Conclusions. Further observations of the detected sources are necessary to clearly determine the origin of the X-ray emission. Moreover, additional objects should be subject to targeted X-ray observations in order to achieve better constraints for the magnetic fields around AGB stars.
\end{abstract}

Key words. stars: AGB and post-AGB - stars: magnetic field - X-rays: stars

\section{Introduction}

The required conditions for asymptotic giant branch (AGB) stars to evolve into planetary nebulae is still a matter of debate. Since AGB circumstellar envelopes (CSEs) are normally spherically symmetric (Castro-Carrizo et al. 2010) and PNe are not (Parker et al. 2006), the shaping during the transition is one of the open questions. One suggested shaping agent is a largescale, circumstellar magnetic field. Circular polarization due to magnetic-field-induced Zeeman splitting has been measured using maser polarization observations (e.g., Herpin et al. 2006; Vlemmings et al. 2005; Bains et al. 2003). For C-type (C/O > 1) stars, Zeeman splitting of the paramagnetic molecule $\mathrm{CN}$ has been used to estimate the magnetic field (Herpin et al. 2009), and recently, the first estimate of a magnetic field around an evolved star using polarized $\mathrm{CO}$ emission was published (Vlemmings et al. 2012). The current measurements are consistent with either an $R^{-1}$ (toroidal) or an $R^{-2}$ (poloidal) dependence, indicating field strengths of order several tens of Gauss at the stellar surface (Vlemmings 2011).

A large-scale magnetic field will likely lead to X-ray emission (Blackman et al. 2001; Pevtsov et al. 2003). However, a large fraction, if not all, of this emission might be absorbed by the high-density wind surrounding the star (Kastner \& Soker 2004a). Targeted X-ray observations have to our knowledge only been performed for three AGB stars: $o$ Cet (Mira), T Cas, and

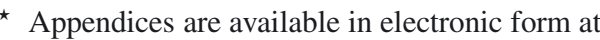
http://www . aanda.org
}

TX Cam. Known symbiotic systems with AGB primaries, e.g., $\mathrm{R}$ Aqr and $\mathrm{CH}$ Cyg, have also been observed (Kellogg et al. 2001; Karovska et al. 2007). Kastner \& Soker (2004b) observed the Mira AB system with XMM and conclude that the weak, predominantely soft $(<3 \mathrm{keV}) \mathrm{X}$-ray emission probably originates from the accretion from Mira A to B, or from coronal activity on Mira B. Later Karovska et al. (2005) reported emission at both binary components, and the detection of a large X-ray outburst on Mira A, with CXO. The spectrum was dominated by a soft $(0.2-0.7 \mathrm{keV})$ component originating from Mira A. The total luminosity was roughly a factor of 2 to 4 higher than that of the previous observations. T Cas and TX Cam were both observed with XMM, but neither was detected (Kastner \& Soker 2004a), possibly due to the absorption of the high density wind.

We have searched the ROSAT, Chandra X-ray Observatory (CXO), and XMM-Newton archives for X-ray sources among a sample of $\sim 500$ well-studied, nearby AGB stars and will report our findings here. In Sect. 2 the sample is presented and the observations are described. The results are given in Sect. 3 and discussed in Sect. 4. Finally, we draw our conclusions in Sect. 5.

\section{Data and analysis}

\subsection{The sample}

We have searched the archives for X-ray detections associated with the 180 AGB stars in the samples of Schöier \& Olofsson (2001), González Delgado et al. (2003), and Ramstedt et al. (2006). The C-type star sample is complete, or close to complete, 
Table 1. AGB stars discussed in detail in the paper.

\begin{tabular}{lcrccr}
\hline \hline Source & $\begin{array}{c}\text { Sp. } \\
\text { type }\end{array}$ & $\begin{array}{c}\text { Var. } \\
\text { type }\end{array}$ & $\begin{array}{c}P^{1} \\
{[\mathrm{~d}]}\end{array}$ & $\begin{array}{c}L^{2} \\
{\left[L_{\odot}\right]}\end{array}$ & \multicolumn{1}{c}{$\begin{array}{c}D^{a} \\
{[\mathrm{pc}]}\end{array}$} \\
\hline UX Ara & $\ldots$ & $\mathrm{M}$ & 250 & 4570 & $1000:$ \\
T Cas & $\mathrm{M}$ & $\mathrm{M}$ & 445 & 9200 & $290^{3}$ \\
TX Cam & $\mathrm{M}$ & $\mathrm{M}$ & 557 & 11900 & $440^{4}$ \\
$o$ Cet & $\mathrm{M}$ & $\mathrm{M}$ & 332 & 6430 & $92^{5}$ \\
W Cyg & $\mathrm{M}$ & $\mathrm{SRb}$ & 131 & $\ldots$ & $173^{5}$ \\
R Dor & $\mathrm{M}$ & $\mathrm{SRb}$ & 338 & $\ldots$ & $55^{5}$ \\
T Dra & $\mathrm{C}$ & $\mathrm{M}$ & 422 & 6550 & $610^{6}$ \\
RT Eri & $\mathrm{M}$ & $\mathrm{M}$ & 371 & 7380 & $277^{7}$ \\
R Leo & $\mathrm{M}$ & $\mathrm{M}$ & 310 & 5920 & $71^{5}$ \\
L Pup & $\mathrm{M}$ & $\mathrm{SRb}$ & 141 & $\ldots$ & $64^{5}$ \\
RW Sco & $\mathrm{M}$ & $\mathrm{M}$ & 388 & 7800 & $870:$ \\
R UMa & $\mathrm{M}$ & $\mathrm{M}$ & 302 & 5750 & $415^{5}$ \\
SS Vir & $\mathrm{C}$ & $\mathrm{SRa}$ & 364 & $\ldots$ & $540^{6}$ \\
\hline
\end{tabular}

Notes. ${ }^{(a)}$ When a reliable parallax $(p / \Delta p>2)$ is available, it has been used to estimate the distance. For the remaining objects we have estimated the distance using the $K$-band magnitude (Skrutskie et al. 2006) and PL relation (Whitelock et al. 2008) (marked by a colon).

References. (1) Samus et al. (2009); (2) Whitelock et al. (1994); Groenewegen \& Whitelock (1996); ${ }^{(3)}$ Loup et al. (1993); ${ }^{(4)}$ Ramstedt et al. (2008); ${ }^{(5)}$ van Leeuwen (2007); ${ }^{(6)}$ Schöier \& Olofsson (2001); (7) Knapp et al. (2003).

out to $500 \mathrm{pc}$. The S-type $(\mathrm{C} / \mathrm{O} \sim 1)$ star sample is a close to complete sample of mass-losing S-type stars out to $600 \mathrm{pc}$. The completeness of the M-type $(\mathrm{C} / \mathrm{O}<1)$ sample has not been thoroughly investigated. To supplement the samples, we added nine stars found to have far-UV excess with GALEX (Sahai et al. 2008) and 291 M-type Miras of Little-Marenin \& Little (1990). Thirteen sample stars were found to have either serendipitous or targeted observations in the archives. These stars are discussed in detail in the paper, and are presented in Table 1 with basic stellar parameters.

\subsection{ROSAT observations}

\subsubsection{Data description and preparation}

Among the AGB star sample listed in Table 1, only 3 objects T Dra, R UMa, and $o$ Cet (Mira) - have spatially coincident $\mathrm{X}$-ray sources listed in the ROSAT source catalogs maintained at HEASARC ${ }^{1}$ (Table 2). X-ray sources very near the positions of $\mathrm{T}$ Dra and R UMa were detected by the ROSAT position sensitive proportional counter (PSPC, in scanning mode) during the ROSAT All-Sky Survey (RASS). These sources are $\sim 8^{\prime \prime}$ and $\sim 2$ " from the catalogued positions of T Dra and R UMa, respectively, and hence within the $10^{\prime \prime}$ positional error. The pointed ROSAT archival observation of Mira (Soker \& Kastner 2003) is also included in the following analysis.

New calibration and analysis (Sect. 2.2.2) were applied to the ROSAT archival observations. The newly determined background-subtracted count rates are 20.6 counts ks$^{-1}$ for T Dra, 39.7 counts ks ${ }^{-1}$ for R UMa, and 6.7 counts ks ${ }^{-1}$ for Mira. According to the RASS faint source catalogue (FSC), the extent of the X-ray emission from $\mathrm{T}$ Dra is significantly larger than the PSPC point spread function (PSF), which suggests extended emission or a superposition of 2 or more X-ray point sources.

To further examine the fields containing T Dra and R UMa, the available GALEX observations (Sahai et al. 2008) were

\footnotetext{
1 http://heasarc.nasa.gov/
}

Table 2. Observations of the sample objects.

\begin{tabular}{lccccr}
\hline \hline Object & Obs. $^{1}$ & ObsID & Obs. Date & $t_{\exp }(\mathrm{ks})$ & $\theta\left(^{\prime}\right)^{2}$ \\
\hline UX Ara & XMM-S & 0306171201 & $2006-03-01$ & 6.1 & 13.2 \\
T Cas & XMM-T & 0148500501 & $2003-02-06$ & 13.2 & 1.1 \\
TX Cam & XMM-T & 0148500101 & $2003-09-04$ & 13.9 & 1.1 \\
$o$ Cet & ROSAT & 201501 & $1993-07-15$ & 9.1 & 0.3 \\
W Cyg & XMMSS & 9146000003 & $2007-11-29$ & $\sim 10^{-2}$ & 0.1 \\
R Dor & XMMSS & 9115800003 & $2006-04-06$ & $\sim 10^{-2}$ & 0.2 \\
T Dra & RASS & 930730 & $1990-07-30$ & 3.0 & 0.1 \\
RT Eri & CXO-S & 4064 & $2003-06-17$ & 4.4 & 1.8 \\
R Leo & XMMSS & 9126500005 & $2006-11-06$ & $\sim 10^{-2}$ & $<0.1$ \\
L Pup & XMMSS & 9077600003 & $2004-03-05$ & $\sim 10^{-2}$ & $<0.1$ \\
RW Sco & XMM-S & 0554440101 & $2008-08-26$ & 30.1 & 9.1 \\
R UMa & RASS & 930513 & $1990-10-05$ & 0.5 & $<0.1$ \\
SS Vir & XMM-S & 0303560101 & $2005-07-10$ & 4.4 & 13.8 \\
\hline
\end{tabular}

Notes. ${ }^{(1)}$ The XMM slew survey is denoted XMMSS. Whether the observations were serendipitous (S) or targeted (T) is also indicated. (2) $\theta$ is the off-axis angle of the object with respect to the aimpoint of the observation.

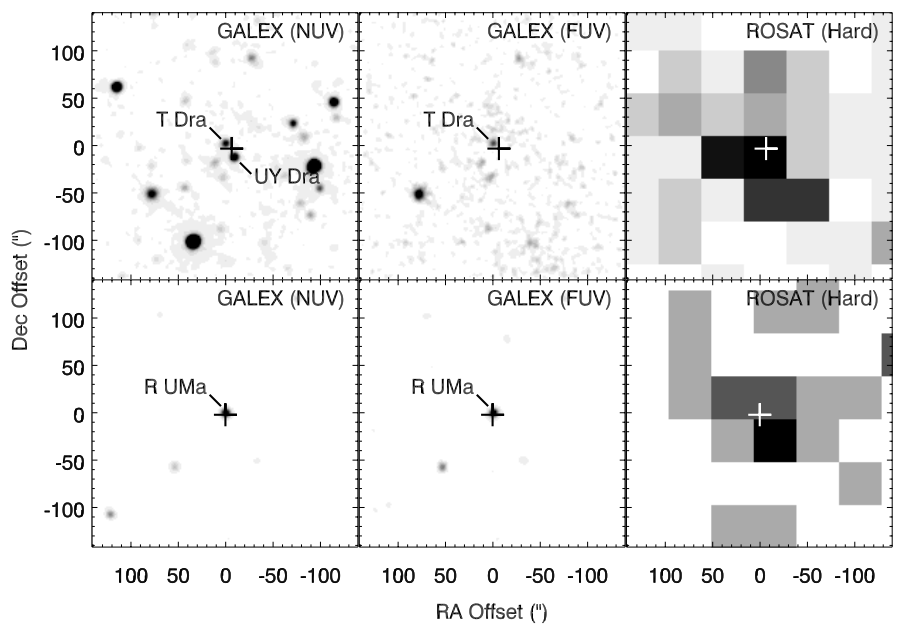

Fig. 1. GALEX NUV (1771-2831 A, left) and FUV (1344-1786 ̊, middle), together with the ROSAT X-ray images (right) of T Dra (upper panel) and R UMa (lower panel). The crosses correspond to the coordinates of the X-ray sources according to the RASS.

obtained from the GALEX archive. The ROSAT X-ray images are presented in Fig. 1 together with the UV images ${ }^{2}$. UY Dra (spectral type K2III-IV, Samus et al. 2009), also seen in the NUV image in Fig. 1 (upper panel, right), is located less than $20^{\prime \prime}$ south-west of T Dra. Although UY Dra is the less likely X-ray source (since it's not visible in the FUV image), new X-ray observations at higher sensitivity and spatial resolution are required to draw firm conclusions as to whether T Dra or UY Dra, or possibly both, is emitting X-rays.

\subsubsection{Spectral analysis}

Source and background spectra were extracted from the ROSAT observations. The exposure times for the spectra are adjusted according to the observation-specific exposure maps. New ancillary response files were created with the HEASoft tool pcarf using the canned response matrix file appropriate for these PSPC observations. Bad channels were removed, and the

2 The spatial resolution of GALEX is 4.3" and 5.3" in the FUV and NUV, respectively, (Morrissey et al. 2007). 
remaining channels are grouped to increase the signal to noise. Fitting of the background-subtracted spectra was performed in XSPEC (version 12.6.0q). Churazov weighting of the spectral bins was used to improve the fit statistics. An absorbed opticallythin thermal plasma (wabs*(raymond)) was assumed as the spectral model. The column density, $N_{\mathrm{H}}$, is used to estimate the absorption due to $\mathrm{H}, \mathrm{H}_{2}$ (assuming equal amounts of atomic and molecular hydrogen), and other atomic species, assuming an optically thin thermal plasma with solar abundances. The low count spectra and ROSAT sensitivity do not allow for the determination of the absorbing column density; instead a range of values of $N_{\mathrm{H}}$ was assumed while the plasma temperature and luminosity were allowed to vary during the fitting procedure.

\subsection{Pointed XMM and CXO observations}

In addition to XMM and CXO observations targeting $\mathrm{T}$ Cas, TX Cam, and Mira (Kastner \& Soker 2004a,b; Karovska et al. 2005), four sample objects have been serendipitously observed in pointed observations by these two observatories. UX Ara, RT Eri, and RW Sco are not detected in these observations. SS Vir is $13.3^{\prime}$ off-axis in its serendipitous XMM observation and falls on the buffer region removed during pipeline processing. In the unfiltered observation, there is apparently a tentative detection at the position of SS Vir. In the case of the pointed XMM observation of T Cas, determined to be an X-ray nondetection by Kastner \& Soker (2004a), a closer inspection of the data showed that a potential X-ray source is marginally detected in one out of the three XMM detectors. However, we conclude that the apparent detections of SS Vir and T Cas are likely due to optical loading and are hence both spurious (see Appendix A).

\subsection{XMM slew observations}

Four sources in the XMM slew survey appear to be associated with AGB stars: R Dor, W Cyg, R Leo, and $\mathrm{L}_{2}$ Pup. They are free of quality warning flags and appear only in the soft $(0.2-2 \mathrm{keV})$ energy band. The raw data was reprocessed and the examination of the spectra and event pattern distributions, together with the visual brightness of the sources, lead us to conclude that the detections are false, and due to optical loading (Appendix A).

\section{Results}

\subsection{X-ray spectral distributions and luminosities}

The spectra of T Dra and R UMa are shown in Fig. 2 together with the reanalyzed ROSAT spectrum of Mira and a model assuming $N_{\mathrm{H}}=5 \times 10^{21} \mathrm{~cm}^{-2}$ and $T_{\mathrm{X}} \sim 10^{7} \mathrm{~K}$. The spectra are not very sensitive to the assumed values and the overlaid models should therefore be considered only as representative. The error bars are weighted Poisson errors. The observed emission peaks at around $1 \mathrm{keV}$ in all three sources.

The fits to the ROSAT spectra demonstrate the difficulty of simultaneously constraining the temperature and column density for the AGB sources. However, we can use the model predictions to determine the reliability of the fitted parameters. For all values of column density, almost all of the best-fit models produce acceptable fit statistics (reduced $\chi^{2}$ between 0.8 and 1.6), but the temperature is often unconstrained at the $90 \%$ confidence level. Figure 3 displays the best-fit temperatures for each AGB source versus the assumed absorbing column density. For low column densities, the temperature becomes unconstrained, but the

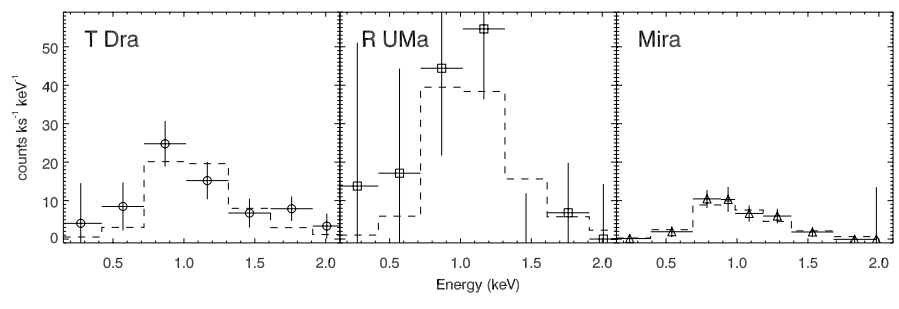

Fig. 2. ROSAT X-ray spectra of T Dra, R UMa, and Mira together with typical model fit (dashed). Mira did not appear in the RASS BSC or FSC, since its count rate is lower than the two FSC objects T Dra and R UMa.

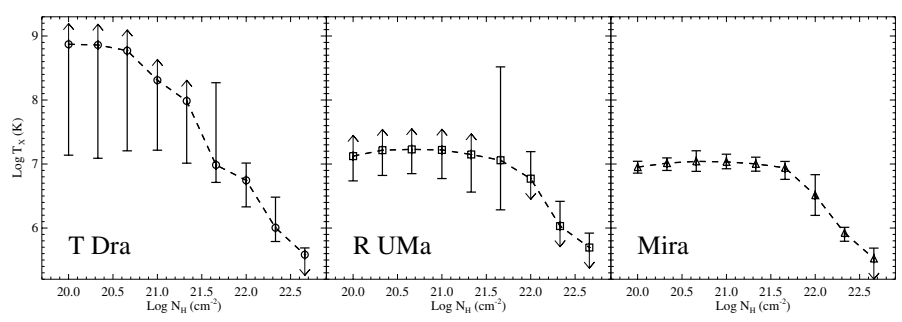

Fig. 3. Best-fit X-ray temperatures as functions of the hydrogen column density for T Dra (squares), R UMa (dots), and Mira (triangles). The $90 \%$ confidence range is indicated by the flat-tipped error bars, while an unconstrained value is indicated by the arrow-tipped error bars. The behavior of the fits reflects the limitations of ROSAT spectra for hard and low count rate sources.
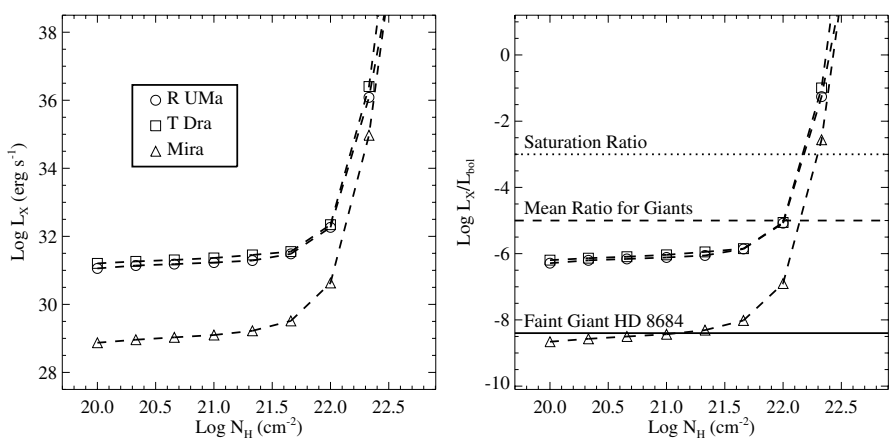

Fig. 4. X-ray luminosities, $L_{\mathrm{X}}$ (left), and $L_{\mathrm{X}} / L_{\text {bol }}$ (right) as functions of $N_{\mathrm{H}}$ for T Dra, R UMa, and Mira. In the right plot, the horizontal lines mark the typical values for coronal saturation around young and active stars $\left(10^{-3}\right.$; Feigelson et al. 2003), the mean activity ratio for G-K giants $\left(10^{-5}\right.$; Gondoin 2005,2007$)$ and the lowest level that has been detected in a first-ascent giant (Hünsch et al. 1996).

lower $90 \%$ confidence range is consistently around $10^{7} \mathrm{~K}$, as expected for coronal or compact object accretion processes.

The unabsorbed source flux is predicted from the model fits with the absorbing column removed, and used along with the distances in Table 1 to determine the X-ray luminosity in the 0.2 to $2.0 \mathrm{keV}$ energy band as a function of the assumed column density (Fig. 4). The X-ray luminosity scaled to the bolometric luminosity of the AGB star provides a strong diagnostic of the physical process responsible for the X-ray emission. These results and their constraints on the column density and origin of the X-ray emission are discussed further in Sect. 4.

\section{Discussion}

\subsection{Estimating the absorbing column density: $N_{H}$}

Based on the luminosity levels shown in Fig. 4, a column density much higher than $10^{22} \mathrm{~cm}^{-2}$ seems unlikely for the sources 
detected by ROSAT. By following Kastner \& Soker (2004a, their Eq. (1)), the hydrogen column density of the circumstellar wind, $N_{\mathrm{H}}$, can be estimated from the mass-loss rate, $\dot{M}$, and the expansion velocity, $v_{\mathrm{e}}$. Using the parameters from Schöier \& Olofsson (2001), the circumstellar hydrogen column density of T Dra is $8.9 \times 10^{22} \mathrm{~cm}^{-2}$. Adopting typical values for M-type stars $\left(\dot{M}=10^{-7} M_{\odot} \mathrm{yr}^{-1}, v_{\mathrm{e}}=7 \mathrm{~km} \mathrm{~s}^{-1}\right)$ yields $N_{\mathrm{H}} \approx 10^{22} \mathrm{~cm}^{-2}$, as an approximate estimate for R UMa. We have compared the results of the equation given by Kastner \& Soker (2004a) to estimates from measurements of the $21 \mathrm{~cm}$-emission of atomic hydrogen around AGB stars (Gérard \& Le Bertre 2006; Matthews et al. 2011) by calculating the hydrogen column densities from the estimated mass and extent of the emission, assuming an $r^{-2}$-density distribution. The results (Appendix B) show no indication that the equation systematically overestimates the hydrogen column density as suggested by Kastner \& Soker (2004a).

If the AGB star is the source of X-ray emission, the detections might indicate that Eq. (1) slightly overestimates $N_{\mathrm{H}}$, but more likely it reflects the simplicity of the analysis. It is to be expected that a process that could be responsible for the $\mathrm{X}$-rays (i.e. binary interaction or a strong magnetic field) would also introduce asymmetries in the circumstellar environment. The emission could therefore emerge from a region where the column density of the AGB envelope falls below a few times $10^{22} \mathrm{~cm}^{-2}$, although the average column density as probed by unresolved observations, is higher. These uncertainties can only be mitigated by additional observations (see Sect. 4.4).

\subsection{X-ray emission from a potential companion}

The suspicion of undetected binary companions to R UMa and T Dra stems from the multiplicity flag ("X") in their HIPPARCos catalog entries. This flag indicates that neither a single or binary astrometric solution could be found and may suggest that the star is a binary (Lindegren et al. 1997). Accretion of the AGB wind onto a compact companion can account for the X-ray emission, e.g., the emission detected from Mira B (Kastner \& Soker 2004b) is believed to be due to accretion onto a compact white dwarf (WD). Furthermore, accretion onto a late-type companion can rejuvenate its corona leading to hard X-ray emission (e.g. Montez et al. 2010; Jeffries \& Stevens 1996).

Sahai et al. (2008) searched for companions to AGB stars with GALEX UV observations in a sample of stars largely selected on the HIPPARCos multiplicity flag. R UMa and T Dra were both found to have UV excesses. Only R UMa was modeled since spectral templates did not exist for carbon-rich AGB stars, e.g. T Dra. Sahai et al. (2008) find that the UV excess of R UMa may be explained by a hot companion at $\sim 9000 \mathrm{~K}$. Their model provides the luminosity relative to the primary star, and assuming a distance to R UMa of $0.5 \mathrm{kpc}$ results in a companion luminosity of $L_{\mathrm{c}}=0.85 L_{\odot}$. This luminosity does not agree with evolutionary tracks for a cooling WD and the origin of the UV excess therefore remains uncertain. The properties determined by Sahai et al. (2008) suggests it is unlikely that accretion onto a WD is responsible for the X-ray emission. Also, in light of these potential X-ray detections, it is possible that the UV excess is unrelated to the photosphere of an unknown companion, but instead due to chromospheric activity around a latetype companion or the AGB star itself. Such an interpretation is consistent with the X-ray emission being caused by coronal emission.

Indeed, the hard X-ray emission detected from WDs is typically attributed to the coronal emission of their late-type main sequence companions (O’Dwyer et al. 2003; Chu et al. 2004; Bilíková et al. 2010). A coarse measure of the spectral distribution is provided by the hardness ratios (HR). The ROSAT PSPC HRs are defined as described in, e.g., Kastner et al. (2003). HR1 is related to the soft part of the spectrum, and a small HR1 indicates a very soft spectrum. HR2 is related to the harder part of the spectrum, and if HR2 is large, the spectrum is hard. The binary WDs detected by ROSAT have consistent HR2 ratios, but only a few reach the high HR1 ratios found from the AGB sources. The HR2 ratios are consistent with high temperature $\left(>10^{6} \mathrm{~K}\right)$ plasma that may be due to coronal or accretion processes. The high HR1 ratios indicate a large absorbing column which attenuates the soft X-ray emission, as expected from the dense AGB mass loss. If we assume that the coronae of unknown companions in T Dra and R UMa are saturated and responsible for the X-ray emission $\left(L_{\mathrm{X}} / L_{\mathrm{bol}} \sim 10^{-3}\right)$, then their luminosities should be $\sim 10 L_{\odot}$ (if $N_{\mathrm{H}}=1 \times 10^{21} \mathrm{~cm}^{-2}$ ). Whereas, if the AGB star is responsible for the X-ray emission (see next section), the $L_{\mathrm{X}} / L_{\text {bol }}$ ratios in Fig. 4 are similar to those observed in late-type giants $\left(10^{-5}\right.$; Gondoin 2005, 2007).

UY Dra could possibly be the source of the X-ray emission in the vicinity of T Dra, but is not visible in the FUV GALEX image whereas T Dra is clearly present. Furthermore, our analysis shows that the extent of the X-ray emission is larger than the ROSAT PSF, perhaps indicating multiple X-ray sources.

\subsection{X-ray emission from the $A G B$ star}

The pulsation-induced shocks above the AGB atmosphere will not emit X-rays. Typical post-shock temperatures are $\lesssim 15000 \mathrm{~K}$ leading to radiative cooling mainly through forbidden lines and hydrogen lines in the optical and UV (Schirrmacher et al. 2003). Thus, if a single AGB star is the source of X-ray emission, one possible explanation is coronal emission from a largescale magnetic field. The origin and feasibility of a large-scale magnetic field on an AGB star is discussed in several papers (e.g., Nordhaus et al. 2007; García-Segura et al. 2005; Soker \& Kastner 2003) and could be due to a dynamo (Blackman et al. 2001), the movement of ionized gas in large convective cells (Hünsch \& Schröder 1996), or the presence of a binary companion as in the cool components of symbiotic systems (Soker 2002; Zamanov et al. 2008).

Pevtsov et al. (2003) found a correlation between magnetic flux and $L_{\mathrm{X}}$ using observations of the Sun and magnetically active stars. Assuming $N_{\mathrm{H}}=1 \times 10^{21}\left(10^{22}\right) \mathrm{cm}^{-2}$, the magnetic flux would be $\approx 10^{23}\left(10^{24}\right) \mathrm{G} \mathrm{m}^{2}$. If we assume a stellar radius of $1 \mathrm{AU}$ and that the X-rays are emitted evenly across the surface, this gives a surface magnetic field of $\sim 1 \mathrm{G}(\sim 10 \mathrm{G})$, which agrees with typical values extrapolated from maser polarimetry measurements of other AGB stars (e.g., Vlemmings 2011).

\subsection{Questions to be addressed by future observations}

The uncertainties related to the absorption of the X-ray emission are many and due to uncertainties in the composition, the atomic/molecular fraction, the dust-to-gas ratio, as well as the effects of circumstellar asymmetries. Spatially resolved observations of the gas and dust distribution around the X-ray detected sources would be needed to evaluate if the detections are the result of less absorption along the line of sight to these sources.

Further X-ray observations are necessary to place meaningful limits on magnetic fields around AGB stars. In Fig. 5 we show the detection thresholds (contours at $\log \left(F_{\mathrm{X}}\right)$ ) for 


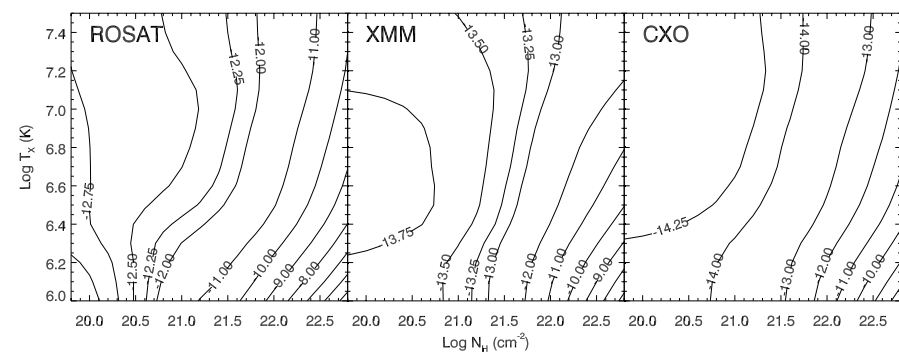

Fig. 5. Detection thresholds $\left(\log \left(F_{\mathrm{X}}\right)\right.$ in $\left.\mathrm{erg} \mathrm{cm}^{-2} \mathrm{~s}^{-1}\right)$ for combinations of plasma temperatures and $N_{\mathrm{H}}$ for ROSAT, XMM, and CXO.

combinations of plasma temperatures and $N_{\mathrm{H}}$ expected from coronal or accretion-related emission from AGB stars or their companions for ROSAT, XMM, and CXO. The detection threshold is determined from the count rates of 20 counts ks$^{-1}$ for ROSAT, 10 counts ks ${ }^{-1}$ for XMM (thick filter), and 1 count $\mathrm{ks}^{-1}$ for CXO. The flux levels of $\mathrm{T}$ Dra and $\mathrm{R}$ UMa are $F_{\mathrm{X}} \approx 10^{-12} \mathrm{erg} \mathrm{cm}^{-2} \mathrm{~s}^{-1}$, and from Fig. 5 it is clear that with $\mathrm{CXO}$ we can detect emission at this level even if it is heavily absorbed, i.e. $N_{\mathrm{H}}>10^{22} \mathrm{~cm}^{-2}$.

\section{Conclusions}

We have searched the ROSAT, XMM, and CXO archives for X-ray detections of a large sample of Galactic AGB stars. Thirteen observations of AGB stars are found. The targeted observations of TX Cam, T Cas, and Mira have previously been analyzed and discussed in Kastner \& Soker (2004a, TX Cam and T Cas), and Karovska et al. (2005, Mira). Four AGB stars, W Cyg, R Dor, R Leo, and $\mathrm{L}_{2}$ Pup were apparently detected as part of the XMMSS, however, the analysis of the data shows that the detected counts are likely due to optical photons (Appendix A). We urge caution when considering visually bright and very red sources discovered in the XMM Slew Survey catalogs. Four additional AGB stars, UX Ara, RT Eri, RW Sco, and SS Vir, were observed serendipitously by XMM or CXO. Three were not detected, and a closer inspection of the data on SS Vir showed that the detection is due to optical loading (Appendix A).

We finally conclude that two of the sample AGB stars, the carbon star T Dra and the M-type star R UMa, likely emit $\mathrm{X}$-rays. The spectra of both sources peak at $\approx 1 \mathrm{keV}$, i.e. at the same energy as the ROSAT spectrum of Mira AB. The low count rate and ROSAT sensitivity does not allow for a secure determination of the absorption or the X-ray luminosity, but we find that the emission is consistent either with accretion onto a WD companion or with coronal emission from a magnetic field of the same order of magnitude as has been inferred from maser observations of AGB stars. To better evaluate the origin of the emission, targeted XMM or CXO observations will be necessary.

Acknowledgements. The authors would like to thank the anonymous referee for helpful suggestions that greatly improved the manuscript. S.R. acknowledges support by the Deutsche Forschungsgemeinschaft (DFG) through the Emmy Noether Research grant VL 61/3-1. This research has made use of data and/or software provided by the High Energy Astrophysics Science Archive Research Center (HEASARC), which is a service of the Astrophysics Science Division at NASA/GSFC and the High Energy Astrophysics Division of the Smithsonian Astrophysical Observatory. We acknowledge with thanks the variable star observations from the AAVSO International Database contributed by observers worldwide and used in this research.

\section{References}

Bains, I., Gledhill, T. M., Yates, J. A., \& Richards, A. M. S. 2003, MNRAS, 338, 287

Bilíková, J., Chu, Y.-H., Gruendl, R. A., \& Maddox, L. A. 2010, AJ, 140, 1433

Blackman, E. G., Frank, A., Markiel, J. A., Thomas, J. H., \& Van Horn, H. M. 2001, Nature, 409, 485

Castro-Carrizo, A., Quintana-Lacaci, G., Neri, R., et al. 2010, A\&A, 523, A59

Chu, Y.-H., Guerrero, M. A., Gruendl, R. A., \& Webbink, R. F. 2004, AJ, 127, 477

Feigelson, E. D., Gaffney, III, J. A., Garmire, G., Hillenbrand, L. A., \& Townsley, L. 2003, ApJ, 584, 911

García-Segura, G., López, J. A., \& Franco, J. 2005, ApJ, 618, 919

Gérard, E., \& Le Bertre, T. 2006, AJ, 132, 2566

Gondoin, P. 2005, A\&A, 444, 531

Gondoin, P. 2007, A\&A, 464, 1101

González Delgado, D., Olofsson, H., Kerschbaum, F., et al. 2003, A\&A, 411, 123

Groenewegen, M. A. T., \& Whitelock, P. A. 1996, MNRAS, 281, 1347

Herpin, F., Baudry, A., Thum, C., Morris, D., \& Wiesemeyer, H. 2006, A\&A, 450,667

Herpin, F., Baudy, A., Josselin, E., Thum, C., \& Wiesemeyer, H. 2009, in IAU Symp., 259, 47

Hünsch, M., \& Schröder, K.-P. 1996, A\&A, 309, L51

Hünsch, M., Schmitt, J. H. M. M., Schröder, K.-P., \& Reimers, D. 1996, A\&A, 310,801

Jeffries, R. D., \& Stevens, I. R. 1996, MNRAS, 279, 180

Karovska, M., Schlegel, E., Hack, W., Raymond, J. C., \& Wood, B. E. 2005, ApJ, 623, L137

Karovska, M., Carilli, C. L., Raymond, J. C., \& Mattei, J. A. 2007, ApJ, 661, 1048

Kastner, J. H., \& Soker, N. 2004a, ApJ, 608, 978

Kastner, J. H., \& Soker, N. 2004b, ApJ, 616, 1188

Kastner, J. H., Crigger, L., Rich, M., \& Weintraub, D. A. 2003, ApJ, 585, 878

Kellogg, E., Pedelty, J. A., \& Lyon, R. G. 2001, ApJ, 563, L151

Knapp, G. R., Pourbaix, D., Platais, I., \& Jorissen, A. 2003, A\&A, 403, 993

Lawson, W. A., Cotrell, P. L., \& Bateson, F. M. 1990, J. Am. Association of Variable Star Observers (JAAVSO), 19, 33

Lindegren, L., Mignard, F., Söderhjelm, S., et al. 1997, A\&A, 323, L53

Little-Marenin, I. R., \& Little, S. J. 1990, AJ, 99, 1173

Loup, C., Forveille, T., Omont, A., \& Paul, J. F. 1993, A\&AS, 99, 291

Lumb, D. 2000, PHS Tools - EPIC Optical Loading

Matthews, L. D., Libert, Y., Gérard, E., et al. 2011, AJ, 141, 60

Montez, Jr., R., De Marco, O., Kastner, J. H., \& Chu, Y.-H. 2010, ApJ, 721, 1820

Moon, T. T., Otero, S. A., \& Kiss, L. L. 2008, J. Am. Association of Variable Star Observers (JAAVSO), 36, 77

Morrissey, P., Conrow, T., Barlow, T. A., et al. 2007, ApJS, 173, 682

Nordhaus, J., Blackman, E. G., Frank, A., et al. 2007, MNRAS, 376, 599

O’Dwyer, I. J., Chu, Y., Gruendl, R. A., Guerrero, M. A., \& Webbink, R. F. 2003, AJ, 125, 2239

Parker, Q. A., Acker, A., Frew, D. J., et al. 2006, MNRAS, 373, 79

Pevtsov, A. A., Fisher, G. H., Acton, L. W., et al. 2003, ApJ, 598, 1387

Ramstedt, S., Schöier, F. L., Olofsson, H., \& Lundgren, A. A. 2006, A\&A, 454, L103

Ramstedt, S., Schöier, F. L., Olofsson, H., \& Lundgren, A. A. 2008, A\&A, 487, 645

Sahai, R., Findeisen, K., Gil de Paz, A., \& Sánchez Contreras, C. 2008, ApJ, 689, 1274

Samus, N. N., Durlevich, O. V., Kazarovets, E. V., et al. 2009, VizieR Online Data Catalog: B/gcvs

Schirrmacher, V., Woitke, P., \& Sedlmayr, E. 2003, A\&A, 404, 267

Schöier, F. L., \& Olofsson, H. 2001, A\&A, 368, 969

Skrutskie, M. F., Cutri, R. M., Stiening, R., et al. 2006, AJ, 131, 1163

Soker, N. 2002, MNRAS, 337, 1038

Soker, N., \& Kastner, J. H. 2003, ApJ, 592, 498

van Leeuwen, F. 2007, A\&A, 474, 653

Vlemmings, W. H. T. 2011, in Asymmetric Planetary Nebulae 5 Conference, eds. A. Ziljstra, F. Lykou, I. McDonald, \& E. Lagadec

Vlemmings, W. H. T., van Langevelde, H. J., \& Diamond, P. J. 2005, A\&A, 434, 1029

Vlemmings, W. H. T., Ramstedt, S., Rao, R., \& Maercker, M. 2012, A\&A, 540, L3

Whitelock, P., Menzies, J., Feast, M., et al. 1994, MNRAS, 267, 711

Whitelock, P. A., Feast, M. W., \& van Leeuwen, F. 2008, MNRAS, 386, 313

Zamanov, R. K., Bode, M. F., Melo, C. H. F., et al. 2008, MNRAS, 390, 377 
Table A.1. Visual magnitudes, $V$, with standard deviation errors, $\Delta V$, at the time of the XMMSS X-ray observation, from the AAVSO archive.

\begin{tabular}{lcrccc}
\hline \hline Source & JD & $V$ & $\Delta V$ & $\begin{array}{c}\text { O.L. for M0 } \\
{\left[\gamma \text { pix }^{-1} \text { fr. }^{-1}\right]}\end{array}$ & $\begin{array}{c}\text { O.L. for M8 } \\
{\left[\gamma \text { pix }^{-1} \text { fr. }^{-1}\right]}\end{array}$ \\
\hline W Cyg & 2454434.2 & 6.1 & 0.3 & 49 & 335 \\
R Dor & 2453831.9 & 6.1 & 0.2 & 49 & 335 \\
R Leo & 2454045.7 & 10.0 & 0.3 & 1.4 & 9.2 \\
L2 Pup & 2453069.5 & 6.9 & 0.5 & 23 & 160 \\
SS Vir & 2453532.4 & 8.0 & 0.5 & 490 & 3360 \\
\hline
\end{tabular}

Notes. The last two columns give the results of the optical loading calculations (in photons pixel ${ }^{-1}$ frame ${ }^{-1}$ ) for an M0 and an M8 source, respectively (see text for details).

\section{Appendix A: Optical loading of XMM observations}

Optical loading occurs because the EPIC detectors have a nonzero response to optical photons (Lumb 2000). Thus, optical photons from a source may generate electrons that would be confused with the charge clouds generated by X-ray photons. For moderately bright optical sources, the net effect is to increase the apparent energy of detected X-ray events. For bright sources, the optical photons that bombard single or adjacent pixels may register as pseudo X-ray events. Three optical blocking filters (thin, medium, and thick) can be employed to reduce optical loading. The limiting magnitude for each blocking filter depends on the spectrum of the source, with redder sources suffering more from optical loading (Lumb 2000). EPIC-pn slew observations are performed in full frame mode (frame time $0.07 \mathrm{~s}$ ) with the medium optical blocking filter, and hence, for visually bright and red sources, e.g. AGB stars, optical loading represents a potential problem. The medium filter is expected to prevent optical contamination from point sources brighter than $V=6-9$ mag depending on the color of the source.

To evaluate the likelihood of optical loading for the AGB stars in Table A.1, their visual light curves were investigated. Three of the objects found in the XMM slew survey are semi-regular variables (SRb) and one is a Mira (M). All vary up to several magnitudes in the optical during one period. Validated data from the American Association of Variable Star Observers (AAVSO) was used to estimate the visual magnitude of the objects at the time of the X-ray observations. Most of the data available are visual data, i.e. obtained by comparing the brightness of the source to nearby comparison stars by eye. This data has been proven to be accurate within 0.1 mag (Lawson et al. 1990; Moon et al. 2008). To estimate the visual magnitude, $V$, at the time of the X-ray observation, a low order polynomial was fitted (using least-square minimization) to the light curve within $\pm 30 \mathrm{~d}$ of the XMMSS observations. The results are given in Table A. 1 together with the standard deviation, $\Delta V$. Three of the AGB stars are brighter than the optical loading limit of the medium optical blocking filter. Only R Leo is fainter.

Lumb (2000) calculates $V$-band zero magnitude spectral energy distributions (SEDs) for a range of spectral types and convolves the SEDs with a model of the detector/optical blocking filter optical response. With appropriate scaling by the observed source $V$-band magnitude, these estimates are used to determine the potential optical contamination. Using the estimated $V$-band magnitudes, the scaled optical flux (photons pixel ${ }^{-1}$ frame $^{-1}$ ) for each detected source is estimated and presented in Table A.1. Lumb (2000) did not consider SEDs representative of AGB stars, thus, the range determined from the M0 and M8 spectral type calculations in Lumb (2000) should be considered as a crude
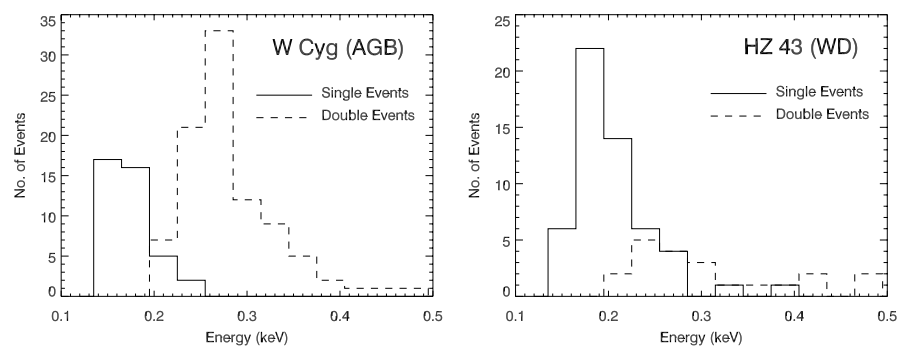

Fig. A.1. Energy distributions of the slew survey-detected sources of the AGB star W Cyg and WD HZ 43. Single events (pattern number 0, solid lines) are the most reliable events in an XMM X-ray source, while double events (pattern numbers $1-4$, dashed lines) are events with charge spread over two pixels. The distribution of single and double events in HZ 43 is the expected distribution for a soft and bright X-ray source, whereas the distributions exhibited by W Cyg and the other AGB stars detected in the slew survey ( $\mathrm{L}_{2}$ Pup, $\mathrm{R}$ Dor, and $\mathrm{R}$ Leo), are indicative of optical loading from these bright red stars.

estimate. The observed objects are likely redder and the optical flux is possibly even higher than estimated from these calculations. If each optically-generated electron contributes a charge of $3.6 \mathrm{eV}$, then a single-pixel pseudo X-ray event of $0.15 \mathrm{keV}$ would be generated from a scaled optical flux of 42 photons pixel $^{-1}$ frame ${ }^{-1}$.

Individually, the brightest stars with $V$-band magnitudes $\sim 6$ have predicted optical fluxes $\left(>42\right.$ photons pixel $^{-1}$ frame $\left.^{-1}\right)$ that are consistent with optically-generated pseudo X-ray events. This suspicion is further confirmed by the energy spectrum for single (pattern 0) and double events (patterns 1-4) depicted in Fig. A.1. These spectra demonstrate that the single pixel events peak at $\sim 0.15 \mathrm{keV}$, while the double events peak around $0.3 \mathrm{keV}$, or twice that of the single events. This behavior is consistent with both piled-up X-ray photons from a high-rate source of soft X-ray photons, and the optically-generated electron clouds of a visually bright source. However, few X-ray sources are as sharply peaked as that of $\mathrm{W}$ Cyg at the energy resolution of $\mathrm{XMM} / \mathrm{EPIC}$, thus the absence of a continuum casts doubt on the veracity of the detected X-ray events. Comparison with the slew detection of the high-rate, soft, X-ray emitting WD, HZ 43 (see Fig. A.1, Slew Survey Source: XMMSL1 J131621.7+290553, ObsID: 9083100004, Obs. Date: 2004-06-23), confirms this suspicion. The single events in the authentic X-ray source associated with HZ 43 appear at higher energies, whereas the pseudo events vanish. We therefore conclude that the XMM slew sources associated with our sample objects are due entirely to optical loading by the visually bright red AGB stars.

\section{A.1. Origin of the $X$-ray emission detected from SS Vir}

The detection of SS Vir is highly suspect because it falls on the edge of the FOV, in a region that is normally discarded from science-quality data products, but which may be exposed. The background-subtracted count rate of SS Vir is $4.8 \pm 1.3$ counts ks ${ }^{-1}$ in the $0.2-2 \mathrm{keV}$ energy range. The soft spectrum of the source is similar to that seen from the slew sources, thus, as described above, the possibility of optical loading of the SS Vir observation must be investigated. The visual magnitude determined from the AAVSO observations of SS Vir at the time of the XMM observation (see Table A.1) suggests SS Vir was clearly bright enough to cause optical loading in the thin optical blocking filter observation. The energy distributions of the high-quality and bad events do not display the high-energy 
tails seen in the optically loaded slew sources (e.g. Fig. A.1); however, the detector optical loading response for bright, red optical sources in this region of the detector is unknown.

\section{Appendix B: Hydrogen column densities estimated from $21 \mathrm{~cm}$-observations}

We have compared the results of the equation given by Kastner \& Soker (2004a) (their Eq. (1)) to estimates from measurements of the $21 \mathrm{~cm}$-emission of atomic hydrogen around AGB stars (Gérard \& Le Bertre 2006; Matthews et al. 2011) by calculating the hydrogen column densities from the estimated mass and extent of the emission, assuming an $r^{-2}$-density distribution (Fig. B.1). Some sources will be missing at the low $N_{\mathrm{H}}$ end of the plot because in low-temperature, high-mass-loss rate AGB stars most of the gas will be in molecular form. Both estimates have substantial uncertainties, and although there is a large scatter around the one-to-one-correlation, there is no indication that the equation systematically overestimates the hydrogen column density as suggested by Kastner \& Soker (2004a).

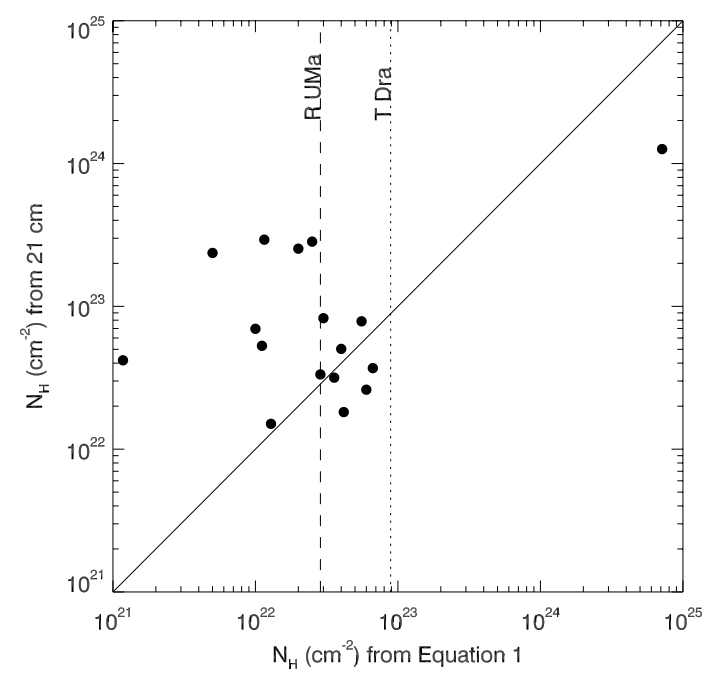

Fig. B.1. Hydrogen column densities estimated from $21 \mathrm{~cm}$-observations (Gérard \& Le Bertre 2006; Matthews et al. 2011) compared to estimates from the mass-loss rate. 